\title{
Basic concepts and recent advancements in penile prosthetic urology
}

Since the invention of the penile prosthesis, decades ago, many manuscripts and journals have been dedicated to the dissemination of research and new information about this sub-section of urology. As the end-point in the management of erectile dysfunction (ED), penile prostheses have evolved from a fringe surgery attempted by only a few into a very effective, safe and common surgical procedure. Over the course of this progression, multiple manuscripts have encompassed similar topics to such a degree that it is now difficult to sort through the mountains of information to identify pertinent information that is critical to the practicing Urologist.

This special edition of Translational Andrology and Urology has been developed with the primary goal of establishing a wide-ranging primer for surgeons who are currently implanting penile prosthetics as well as students and physicians who are looking to get more reliable information and background into current topics.

I sought to start this issue off with a discussion that many Urologists are not too familiar with. Drs. Wassersug and Wibowo gave an excellent summary of the non-pharmacological and non-surgical strategies to help treat ED. Specifically, an in-depth discussion on the use external penile prostheses, penile sleeves, and penile support devices was provided. While not currently a major tool in the armamentarium of the practicing Urologist, it was hoped that this article would bring to light new and innovative treatment strategies. Following this, Dr. Langille and Dr. Bienick respectively described the use of the malleable prostheses both post priapism and following infectious salvage. Dr. Hsieh and colleagues eloquently described the use of penile prosthetic surgery in the management of Peyronie's disease while the accompanying Editorial questioned how Xialfex affected the surgical placement of penile prostheses. Adverse events following prosthetic surgery were summarized in an original paper by Dr. Dabaja who queried the American College of Surgeons database to identify infectious causes as the most common complication immediately post prosthesis insertion.

In an effort to tackle important frontline clinical issues, Dr. Ramasamy was recruited to provide insight into medical preoperative considerations while the accompanying editorial focused on the value of medical pre-assessment in patients due for surgery. Dr. Wosnitzer then nicely summarized post-operative complications and management.

After that, a break from clinical manuscripts allowed contemplation of both the history (via Dr. Pastuszak) and the future (via Dr. Hakky) of the penile prosthesis. Following this, Dr. Coward highlighted pre-operative counseling and expectations while the accompanying Editorial sought to give all readers an example of a thorough informed consent for a penile prosthetic surgery.

To conclude the issue, Dr. Lipshultz detailed the roles of penile prosthetics in the management of ED post cancer therapy and Dr. Smith gave an excellent review of how to troubleshoot intra-operative complications. Lastly, Dr. Kramer was sought to provide a brief communication discussing his novel use of amnion/chorion grafts in penile prosthesis implantation.

As editor of this special issue I would like to give my sincere appreciation to all of the contributors. Each of the authors put in a lot time and effort in order to bring this collection together. This was time that could have been spent with family and friends, but was instead used to help other physicians develop a more thorough understanding of the importance of penile prostheses in our patient's lives. Reading through the manuscripts has expanded my knowledge and the tips, tricks and suggestions provided by these leaders of prosthetic Urology were very informative. I hope that this issue of Translational Andrology and Urology will be one resource that every practicing Urologist will find valuable enough to keep on their shelves as the go-to resource for all issues related to penile prosthetics.

\section{Acknowledgements}

None. 


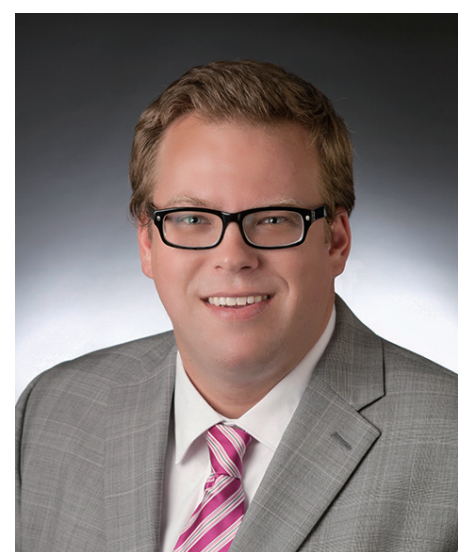

Jason R. Kovac
Dr. Jason R. Kovac, MD, PhD, FACS, FRCSC Men's Health Center, Indianapolis, Indiana 46260, USA. (Email:jkovac@urologyin.com) doi: $10.21037 /$ tau.2017.11.08

Conflicts of Interest: The author has no conflicts of interest to declare. View this article at: http://dx.doi.org/10.21037/tau.2017.11.08

Cite this article as: Kovac JR. Basic concepts and recent advancements in penile prosthetic urology. Transl Androl Urol 2017;6(Suppl 5):S765-S766. doi: 10.21037/tau.2017.11.08 